\title{
NECESSIDADES DE INFORMAÇÃO A RECEPTORES DE TRANSPLANTE DE FÍGADO
}

\author{
Information needs for liver transplantation recipients
}

\author{
Luciana da Costa Ziviani', Karina Dal Sasso Mendes ${ }^{2}$, Tatiane Matins-Pedersoli ${ }^{1}$ \\ Fabiana Murad Rossin Molina ${ }^{3}$, Cristina Maria Galvão²
}

\section{RESUMO}

Objetivo: Avaliar as necessidades de informação de receptores de transplante de fígado em atendimento ambulatorial hospitalar. Método: Estudo descritivo conduzido em centro transplantador brasileiro. A amostra foi composta por 92 participantes. A coleta de dados ocorreu no período de cinco meses, com a aplicação de instrumento adaptado. Resultados: As necessidades de informação que obtiveram médias maiores foram as complicações após o transplante $(5,35)$, os medicamentos após a cirurgia $(5,29)$, e alimentação após o transplante $(4,53)$. As necessidades de informação que obtiveram médias menores foram referentes à gravidez e anticoncepção $(0,28)$, atividades sociais e de lazer $(0,89)$, e retorno ao trabalho/estudos $(0,97)$. Conclusão: O conhecimento produzido sobre as necessidades de informação pode auxiliar no planejamento de intervenções educativas, as quais podem melhorar a qualidade da assistência, a adesão ao tratamento e a prevenção de complicações após o transplante.

Descritores: Enfermagem Perioperatória; Transplante de Fígado; Competência em Informação; Determinação de Necessidades de Cuidados de Saúde; Transplantados.

\footnotetext{
Instituições:

${ }^{1}$ Centro Interescolar do Hospital das Clínicas da Faculdade de Medicina de Ribeirão Preto da Universidade de São Paulo, Ribeirão Preto, São Paulo, Brasil.

${ }^{2}$ Departamento de Enfermagem Geral e Especializada da Escola de Enfermagem de Ribeirão Preto da Universidade de São Paulo, Ribeirão Preto, São Paulo, Brasil.

${ }^{3}$ Comissão de Controle de Infecção Hospitalar do Hospital das Clínicas da Faculdade de Medicina de Ribeirão Preto da Universidade de São Paulo, Ribeirão Preto, São Paulo, Brasil.
}

\section{Correspondência:}

Karina Dal Sasso Mendes

E-mail: dalsasso@eerp.usp.br

https://doi.org/10.53855/bjt.v24i1.002

Recebido em: 21/12/2020

Aceito em: $01 / 03 / 21$

\section{INTRODUÇÃO}

A readaptação do paciente após o transplante de fígado deve iniciar-se na internação e permanecer após a alta hospitalar e no acompanhamento ambulatorial, com o objetivo de promover a independência para os cuidados necessários no domicílio. ${ }^{1}$ Para tal, o ensino é crucial e pode favorecer a adesão do paciente e cuidadores às informações recebidas, no uso dos medicamentos e na redução das complicações e reinternações. ${ }^{2}$ Além disso, o aprendizado do paciente pode auxiliar na promoção e manutenção da saúde, bem como estimular a sua participação no tratamento e na reabilitação. ${ }^{3-4}$

A educação em saúde pode ser definida como o processo que melhora o conhecimento e as habilidades do paciente no manejo do autocuidado, para o comportamento adequado em suas práticas de saúde. ${ }^{5}$ Esse processo envolve a identificação do que ensinar, considerando 
as principais barreiras para o aprendizado, a definição dos objetivos individuais, a escolha das melhores intervenções e a realização de avaliação criteriosa de todo o processo ensino-aprendizagem (E-A). ${ }^{6}$

Para o processo de E-A ser efetivo, é importante a participação do paciente. Esse processo deve ser visto como uma ação conjunta, na qual, por exemplo, o paciente ajuda a determinar quais são as informações que devem ser fornecidas. As intervenções educativas devem ser baseadas no conhecimento científico, na experiência clínica e contemplar as necessidades do paciente. ${ }^{5-6}$

A equipe multiprofissional deve considerar não apenas o que o paciente precisa saber, mas principalmente o que ele deseja saber, suas percepções e expectativas. Os resultados de estudos demonstraram que os pacientes relataram maior satisfação e adesão ao tratamento, redução da ansiedade e melhora na qualidade de vida, quando perceberam que as preocupações e déficits do conhecimento foram abordados pelos profissionais de saúde. ${ }^{4,7-8}$

O ensino do paciente é tópico de interesse entre os enfermeiros, sendo considerado como papel relevante deste profissional. O enfermeiro tem responsabilidade no investimento de esforços em atividades que promovam o aperfeiçoamento de intervenções educativas, com o uso de estratégias de aprendizagem, as quais, podem melhorar a qualidade dos resultados para o paciente e a família. Por meio da educação em saúde, o enfermeiro pode proporcionar ao paciente a sua adaptação à doença, a prevenção de agravos e complicações, bem como a aplicação terapêutica prescrita pelo médico de forma segura e efetiva. , $^{6-9}$

O primeiro passo para o processo E-A é o indivíduo reconhecer quais são as suas necessidades de informação, as quais podem ser definidas como a falta de habilidade, domínio ou conhecimento da pessoa em lidar com aspectos considerados importantes para sua vida. ${ }^{8}$ Conhecer o que o paciente quer e precisa saber é relevante para a evolução do processo E-A, no qual devem ser utilizadas estratégias didáticas voltadas para a participação, vivências e dificuldades, respeitando a individualidade de cada aprendiz. ${ }^{5-6}$

O desenvolvimento do presente estudo consiste no primeiro passo para auxiliar a implementação do processo E-A de forma efetiva nos serviços de saúde. Salienta-se, ainda, a importância desse processo devido à complexidade do procedimento e à necessidade de compreender todas as fases do transplante de fígado, o que requer constante provisão de informações pela equipe multiprofissional para o paciente e a família.
Assim, os resultados da pesquisa podem auxiliar na identificação de problemas relacionados às informações oferecidas no programa de transplante e oferecer subsídios para repensar a atuação do enfermeiro na prática clínica. Além disso, pode fornecer dados que contribuam para a implementação de intervenções educativas efetivas no pós-operatório do transplante de fígado, bem como diminuir as lacunas de conhecimento devido à escassez de estudos na literatura internacional e nacional sobre as necessidades de informação de pacientes submetidos a transplantes de órgãos. Frente ao exposto, a pesquisa teve como objetivo avaliar as necessidades de informação de receptores de transplante de fígado, em atendimento ambulatorial de centro transplantador de referência do interior do estado.

\section{MÉTODOS}

Trata-se de estudo com delineamento de pesquisa não experimental, tipo descritivo. O estudo foi realizado em hospital geral, público, do interior do estado de São Paulo, com programa de transplante de fígado cadastrado no Sistema Nacional de Transplantes. A população foi constituída pelos receptores de fígado, com doador falecido, que estavam em atendimento ambulatorial no ano de condução da pesquisa.

Em relação aos critérios de seleção, foram incluídos os participantes com idade igual ou superior a 18 anos, de ambos os sexos. Foram excluídos os pacientes que apresentaram sequela neurológica de encefalopatia ou com déficit cognitivo e os que faltaram ou não compareceram ao retorno ambulatorial no período da coleta de dados.

No início da coleta de dados, 135 receptores de fígado estavam em atendimento ambulatorial no hospital selecionado para a condução do estudo. Assim, a amostra foi composta de 92 pacientes que atenderam aos critérios de seleção. Dos 135 pacientes, cinco foram excluídos por sequela neurológica de encefalopatia ou déficit cognitivo e os 38 receptores restantes foram excluídos em decorrência da falta à consulta ambulatorial ou não agendamento da mesma, durante o desenvolvimento da pesquisa. A coleta de dados ocorreu no período de cinco meses.

Para a coleta de dados, foi elaborada versão adaptada de instrumento construído e submetido à validação aparente e de conteúdo por pesquisadores nacionais na temática enfermagem em transplante de fígado. ${ }^{4} \mathrm{~A}$ versão adaptada do instrumento também foi submetida à validação aparente e de conteúdo por cinco juízes (três enfermeiros e dois médicos). A concordância das respostas entre os juízes sobre os itens do instrumento 
foi superior a $80 \%$. As sugestões apresentadas foram referentes à forma de apresentação do instrumento, as quais foram acatadas pelos pesquisadores. Assim, o instrumento empregado na coleta de dados tinha três partes. Na primeira, os dados de registro eram direcionados para a identificação sociodemográfica do paciente (dados de identificação). Na segunda parte, os dados eram referentes ao período pós-operatório de transplante de fígado e, na terceira parte, sobre as necessidades de informação para os receptores de transplante de fígado.

A coleta de dados foi realizada da seguinte forma: (1) no início de cada semana, durante o período de coleta de dados, o pesquisador tinha conhecimento da listagem de receptores agendados para a sextafeira, por meio do sistema informatizado do hospital; (2) no dia agendado para o paciente, antes da consulta médica, o pesquisador forneceu as informações quanto ao objetivo da pesquisa, convidando-o para participar do estudo e, por meio de entrevista, realizou a aplicação do instrumento de coleta de dados; (3) em relação à aplicação da terceira parte do instrumento (necessidades de informação), o paciente foi motivado a verbalizar o que gostaria de aprender. Para tal, foi formulado um cartão, contendo 22 sugestões de assuntos relacionados ao pós-operatório de transplante de fígado contidas no instrumento, ficando a critério do paciente utilizar ou não o cartão mencionado. Cada paciente elencou 10 assuntos; (4) após a entrevista, os dados não informados pelo paciente foram coletados do prontuário e do site da Central Estadual de Transplantes.

Para compreender a necessidade de informação dos receptores de transplante de fígado, as 22 sugestões de assuntos foram agrupadas em cinco categorias preconizadas por estudiosos ${ }^{5}$ sobre os principais assuntos a serem abordados no preparo de receptores de transplante de órgãos para o autocuidado no domicílio, a saber: (1) manejo das potenciais complicações (complicações em longo prazo, higiene corporal, exposição solar, alimentação, recidiva da doença, vacinação); (2) manejo dos medicamentos; (3) questões sobre estilo de vida (exercícios físicos, atividade sexual, métodos anticoncepcionais e gravidez, retorno ao trabalho, estudo, uso de substâncias alcoólicas e tabaco, atividades sociais e de lazer); (4) práticas de autocuidado (cuidados com a ferida cirúrgica, drenos e cateteres, autocuidado no domicílio, alterações emocionais, uso de máscara após a cirurgia); e (5) seguimento após alta hospitalar (realização de exames clínicos e laboratoriais, seguimento às consultas ambulatoriais, contato com a família doadora, sobrevida após o transplante).

A análise dos dados foi realizada na forma descritiva.
Os dados foram inseridos em planilha eletrônica do programa Excel®. Após o processo de validação para correção de possíveis erros de digitação, o banco de dados foi exportado para o programa estatístico GraphPad InStat 3.05 (GraphPad Software, Inc) e a estatística descritiva foi executada, tais como: média aritmética, desvio-padrão, mediana, valores mínimo e máximo para as variáveis quantitativas. As variáveis qualitativas investigadas foram apresentadas pela frequência de distribuição dos participantes entre as categorias existentes.

Para o cálculo das necessidades de informação, cada paciente elencou 10 assuntos, sendo que as respostas obtidas foram listadas em ordem decrescente de importância, ou seja, o tópico de maior interesse para o participante recebeu a pontuação 10 e o de menor interesse recebeu um ponto. Desta forma, para cada uma das sugestões de assunto consideradas, realizou-se a média aritmética por meio da divisão entre a somatória de cada sugestão e o número total de indivíduos participantes $(n=92)$. Para salientar o interesse dos participantes em relação a cada sugestão de assunto apresentada, também foi indicado o número de pacientes que responderam a cada sugestão investigada no estudo.

Para a comparação de dados de determinada variável quantitativa entre dois grupos, inicialmente, verificou-se a distribuição normal por meio do teste de normalidade de Kolmogorov-Smirnov. Caso os grupos amostrais apresentassem distribuição normal ( $p>0,05)$, o método estatístico paramétrico foi empregado (teste $t$ de Student), para comparações entre as médias de duas amostras independentes. Caso ao menos um dos grupos amostrais apresentasse desvios significativos em relação à distribuição normal $(p<0,05)$, empregou-se o teste $U$ de Wilcoxon-Mann-Whitney, método estatístico não paramétrico para comparações entre as médias de duas amostras independentes. O nível de significância adotado foi de $\alpha=0,05$.

A pesquisa foi aprovada pelo Comitê de Ética em Pesquisa da instituição (CAE no 20115713.3.0000.5393), em conformidade com a Resolução n. 466/2012, do Conselho Nacional de Saúde.

\section{RESULTADOS}

Dentre as características da amostra, houve predomínio do sexo masculino $(n=71 ; 77,17 \%)$. A média de idade foi de 54,48 anos (DP=10,21), a maioria apresentava idade superior a 51 anos, com 39 (42,39\%) receptores com idade entre 51 e 59 anos e 29 (31,52\%) com idade superior a 60 anos, sendo os valores mínimo e máximo 
de 21 e 75 anos. Na análise do grau de instrução dos pacientes (anos de estudos), a média foi de 9,02 anos $(\mathrm{DP}=4,24)$, destaca-se que $50 \%$ cursaram até o ensino fundamental.

Em relação ao perfil clínico, no dia do transplante, os pacientes apresentaram média de 24,34 pontos $(\mathrm{DP}=4,98)$ nos valores do Model for End-stage Liver Disease (MELD), sendo que a maioria $(n=67 ; 72,83 \%)$ foi classificada entre 20 e 29 pontos, considerado grau moderado de severidade da doença hepática. No presente estudo, 52 (56,52\%) pacientes tiveram situação especial para a realização da cirurgia, ou seja, apresentaram condições clínicas que aumentaram o valor do MELD, tais como hepatocarcinoma, ascite refratária, encefalopatia hepática, entre outras.

No que tange à causa da doença crônica do fígado que acarretou o transplante, constatou-se predomínio da causa viral (hepatites B ou C) em 38 (41,30\%) pacientes da amostra, seguida de causa alcoólica em $22(23,91 \%)$, causa criptogenética em $15(16,30 \%)$ e quatro $(4,35 \%)$ com causa viral e alcoólica em conjunto. Dos 92 participantes, independentemente da etiologia que acarretou a doença crônica do fígado, 28 (30,43\%) apresentaram hepatocarcinoma durante o período de espera para o transplante de fígado.

$\mathrm{Na}$ análise do período de internação após o transplante, a média foi de 21,35 dias ( $D P=19,61)$, valores mínimo e máximo de cinco e 120 dias de internação. Dos 92 participantes, $36(39,13 \%)$ foram classificados no intervalo de sete a 14 dias de internação, 29 (31,52\%), com internação acima de 21 dias, 18 (19,57\%) de 15 a 21 dias, e apenas nove $(9,78)$ ficaram internados por até sete dias.

$\mathrm{Na}$ amostra investigada, $44(47,83 \%)$ pacientes não tiveram complicações no período pós-operatório, sendo que, dos 48 que apresentaram complicações após o transplante, $33(68,75 \%)$ foram complicações clínicas e 15 (31,25\%), cirúrgicas. As complicações cirúrgicas incluíram as vasculares, biliares, sangramentos cirúrgicos e lesões no diafragma, e as complicações clínicas foram a rejeição do enxerto, lesões de preservação do órgão transplantado, infecções, toxicidade aos medicamentos, sangramentos por coagulopatias e comprometimento da função renal. Salienta-se que, dos 92 participantes da pesquisa, apenas cinco $(5,43 \%)$ foram submetidos ao retransplante devido às complicações pós-operatórias.

Com relação ao Índice de Massa Corporal (IMC), houve predomínio de pacientes com peso normal, ou seja, 42 (45,65\%) apresentaram IMC entre 18,50 e $24,99 \mathrm{Kg} /$ m2 após o transplante, seguidos de $31(33,70 \%)$ com sobrepeso (IMC acima de $25 \mathrm{Kg} / \mathrm{m} 2$ ). Quanto à terapia medicamentosa de imunossupressão empregada para evitar rejeição ao órgão transplantado, 34 (36,96\%) participantes fizeram uso apenas do tacrolimus, 35 (38,04\%) utilizaram o tacrolimus associado ao micofenolato sódico, $10(10,87 \%)$ utilizaram ciclosporina associada ao micofenolato sódico, cinco $(5,43 \%)$ fizeram uso apenas de ciclosporina e oito $(8,70 \%)$ utilizavam outros medicamentos imunossupressores.

No que se refere às fontes de informações relacionadas às necessidades de aprendizagem dos receptores de transplante de fígado, 91 (98,91\%) pacientes referiram ter recebido as informações necessárias para os cuidados domiciliares após o transplante, no momento da alta hospitalar, sendo que $90(97,83 \%)$ citaram a equipe multidisciplinar como uma das fontes principais de informação no pós-operatório. Em relação à preferência sobre os métodos de ensino na aquisição de conhecimentos, a leitura $(60,87 \%)$, a orientação individual $(54,35 \%)$ e ouvir explicações $(52,17 \%)$ foram as estratégias verbalizadas com maior frequência pelos receptores de transplante de fígado. Ressalta-se que os pacientes poderiam optar por até três métodos de ensino nas respostas ao item.

$\mathrm{Na}$ análise da média das necessidades de informação, as complicações após o transplante foi o assunto que obteve média maior $(5,35)$ e, portanto, pode-se inferir de maior interesse para os receptores de transplante de fígado (Tabela 1). Outra necessidade em destaque foi sobre os medicamentos após a cirurgia (efeitos colaterais e cuidados), a qual teve média de 5,29, seguida pela alimentação após o transplante (média de 4,53). As necessidades de informação de menor interesse foram referentes à gravidez e anticoncepção (média de 0,28 ), atividades sociais e de lazer (média de 0,89 ) e ao retorno ao trabalho/estudos (média de $0,97)$.

$\mathrm{Na}$ comparação de resultados, os receptores de fígado foram divididos em dois grupos: grupo I $(n=46)$, pacientes com escolaridade igual ou inferior ao ensino fundamental, e o grupo II $(n=46)$ pacientes com escolaridade igual ou superior ao ensino médio (Tabela 2). $\mathrm{Na}$ análise estatística, os resultados evidenciaram que a idade $(p<0,0001)$ e a média de anos de estudos $(p=0,0088)$ entre os grupos apresentaram diferença estatisticamente significante. Em relação às necessidades de informação, as médias foram semelhantes (sem diferença estatisticamente significante) entre os grupos, nas cinco categorias sobre sugestões de assunto, sendo que a média maior foi na categoria manejo dos medicamentos para ambos os grupos (grupo $I=5,28$ e grupo $\|=5,30$ ). 
Tabela 1 - Distribuição dos valores das respostas sobre as necessidades de informação dos receptores de transplante de fígado.

\begin{tabular}{|c|c|c|c|}
\hline Necessidades de informação & $\begin{array}{c}\text { Classificação } \\
\text { por importância }\end{array}$ & $\begin{array}{l}\text { № de pacientes } \\
\text { respondentes }\end{array}$ & Média \\
\hline \multicolumn{4}{|l|}{ Manejo das potenciais complicações } \\
\hline Complicações após o TF* & 492 & 62 & 5,35 \\
\hline Higiene corporal e bucal & 399 & 59 & 4,34 \\
\hline Exposição solar & 305 & 58 & 3,32 \\
\hline Alimentação após o TF* & 417 & 74 & 4,53 \\
\hline Recidiva do vírus no fígado & 164 & 39 & 1,78 \\
\hline Vacinas permitidas & 206 & 54 & 2,24 \\
\hline \multicolumn{4}{|l|}{ Manejo dos medicamentos } \\
\hline Medicamentos após o TF* & 487 & 65 & 5,29 \\
\hline \multicolumn{4}{|l|}{ Questões sobre estilo de vida } \\
\hline Exercícios físicos & 187 & 37 & 2,03 \\
\hline Relações sexuais & 128 & 26 & 1,39 \\
\hline Gravidez e anticoncepção & 26 & 4 & 0,28 \\
\hline Retorno ao trabalho/estudo & 89 & 17 & 0,97 \\
\hline Consumo de bebida alcóolica & 237 & 42 & 2,58 \\
\hline Uso de cigarro & 125 & 29 & 1,36 \\
\hline Atividades sociais e de lazer & 82 & 27 & 0,89 \\
\hline \multicolumn{4}{|l|}{ Práticas de autocuidado } \\
\hline Cuidados com a ferida operatória, drenos e sondas & 337 & 45 & 3,66 \\
\hline Autocuidado no domicílio & 148 & 29 & 1,61 \\
\hline Alterações emocionais & 175 & 42 & 1,90 \\
\hline Uso de máscaras após o TF* & 181 & 56 & 1,97 \\
\hline \multicolumn{4}{|l|}{ Seguimento após alta hospitalar } \\
\hline Rotina de coleta de exames & 353 & 59 & 3,84 \\
\hline Seguimento ambulatorial & 292 & 47 & 3,17 \\
\hline Contato com a família doadora & 96 & 18 & 1,04 \\
\hline Tempo de sobrevida após o TF* & 134 & 31 & 1,46 \\
\hline
\end{tabular}

Além da escolaridade, realizou-se a comparação entre os grupos, de acordo com o tempo de transplante, sendo o grupo I $(n=41)$, pacientes com tempo de transplante < três anos, e o grupo II $(n=51)$, pacientes com tempo de transplante $\geq 3$ (Tabela 3 ). A idade $(p=0,0247)$, o MELD $(p<0,0001)$, o IMC $(p=0,0116)$ e o tempo de transplante $(p<0,0001)$ apresentaram diferença estatisticamente significante entre os grupos. Em relação às necessidades de informação, as médias foram semelhantes (sem diferença estatisticamente significante) entre os grupos, em quatro categorias, sendo que houve diferença estatisticamente significante na categoria seguimento após alta hospitalar, com média maior no grupo II $(2,72$ versus 1,95$)$.

\section{DISCUSSÃO}

O conhecimento do perfil sociodemográfico e clínico, bem como o conjunto de necessidades de informação de receptores de fígado são aspectos relevantes para o enfermeiro planejar intervenções educativas efetivas, para contribuir na adesão do paciente ao tratamento proposto e ao sucesso do transplante.

Após o transplante, o paciente passa por diferentes alterações em sua rotina e estilo de vida. Ele precisa realizar retornos ambulatoriais semanais, cuja frequência pode diminuir conforme a evolução da condição de saúde. Além disso, coletas periodicas de exames sanguíneos, ingestão rigorosa de quantidade elevada 
Tabela 2 - Distribuição dos receptores de transplante de fígado, segundo a escolaridade, idade, MELD, índice de massa corporal, período de internação, tempo de transplante e necessidades de informação.

\begin{tabular}{|c|c|c|c|}
\hline & & & \\
\hline & \multicolumn{2}{|c|}{ Receptores $(n=92)$} & \multirow{4}{*}{$\mathbf{p}$} \\
\hline & \multirow{3}{*}{$\begin{array}{l}\text { Grupo I }(n=46) \\
\leq \text { Ensino fundamen-tal } \\
\text { Média (desvio padrão) }\end{array}$} & \multirow{3}{*}{$\begin{array}{c}\text { Grupo II ( } n=46 \text { ) } \\
\geq \text { Ensino médio } \\
\text { Média (desvio padrão) }\end{array}$} & \\
\hline & & & \\
\hline & & & \\
\hline Escolaridade (anos) & $5,24(1,80)$ & $12,09(2,36)$ & $<0,0001 *$ \\
\hline Idade (anos) & $56,96(8,70)$ & $52,00(11,07)$ & $0,0088^{*}$ \\
\hline MELD** (pontos) & $23,49(4,75)$ & $25,12(5,12)$ & $0,0811 *$ \\
\hline Índice de Massa Corporal (Kg/m2) & $26,90(5,09)$ & $25,79(4,32)$ & $0,2641 * * *$ \\
\hline Período de internação (dias) & $22,74(22,45)$ & $19,96(16,42)$ & $0,8117^{*}$ \\
\hline Tempo de transplante (dias) & $1.696,59(1.297,24)$ & $1.321,11(1.192,50)$ & $0,1587^{*}$ \\
\hline \multicolumn{4}{|l|}{ Necessidades de informação (categorias) } \\
\hline Manejo das potenciais complicações & $3,52(1,11)$ & $3,66(0,97)$ & $0,6846^{*}$ \\
\hline Manejo dos medicamentos & $5,28(4,25)$ & $5,30(4,20)$ & $0,7810^{*}$ \\
\hline Questões sobre estilo de vida & $1,32(0,89)$ & $1,39(1,05)$ & $0,9191^{*}$ \\
\hline Práticas de autocuidado & $2,14(1,44)$ & $2,43(1,42)$ & $0,3194^{* * *}$ \\
\hline Seguimento após alta hospitalar & $2,70(1,79)$ & $2,06(1,59)$ & $0,0682 *$ \\
\hline
\end{tabular}

*Teste U de Wilcoxon-Mann-Whitney; $\quad$ ** MELD=Model for End-stage Liver Disease; $\quad$ *** Teste t de Student

Tabela 3 - Distribuição dos receptores de transplante de fígado, segundo a escolaridade, idade, MELD, indice de massa corporal,
periodo de internação, tempo de transplante e necessidades de informação. Ribeirão Preto, SP, Brasil, 2014

Tabela 3 - Distribuição dos receptores de transplante de fígado, segundo a escolaridade, idade, MELD, indice de mas
periodo de internação, tempo de transplante e necessidades de informação. Ribeirão Preto, SP, Brasil, 2014

\section{Receptores ( $\mathrm{n}=92$ )}

\begin{tabular}{|c|c|c|c|}
\hline & & & \\
\hline & $\begin{array}{l}\quad \text { Grupo l }(\mathrm{n}=46) \\
\leq \text { Ensino fundamen-tal } \\
\text { Média (desvio padrão) }\end{array}$ & $\begin{array}{c}\text { Grupo II ( } \mathrm{n=46)} \\
\geq \text { Ensino médio } \\
\text { Média (desvio padrão) }\end{array}$ & $\mathbf{p}$ \\
\hline Escolaridade (anos) & $9,27(3,91)$ & $8,18(4,10)$ & $0,1162 * *$ \\
\hline Idade (anos) & $52,29(9,31)$ & $56,24(10,64)$ & $0,0247 * *$ \\
\hline MELD** (pontos) & $26,54(4,91)$ & $22,15(4,03)$ & $<0,0001 * *$ \\
\hline Índice de Massa Corporal (Kg/m2) & $24,97(4,22)$ & $27,45(4,87)$ & $0,0116 * * * *$ \\
\hline Período de internação (dias) & $19,00(13,55)$ & $23,24(23,34)$ & $0,5689 * *$ \\
\hline Tempo de transplante (dias) & $438,37(303,91)$ & $2.369,43(1.044,16)$ & $<0,0001^{* *}$ \\
\hline \multicolumn{4}{|l|}{ Necessidades de informação (categorias) } \\
\hline Manejo das potenciais complicações & $3,70(0,96)$ & $3,50(1,10)$ & $0,3625 * * * *$ \\
\hline Manejo dos medicamentos & $5,15(4,26)$ & $5,41(4,20)$ & $0,7616 * *$ \\
\hline Questões sobre estilo de vida & $1,46(1,04)$ & $1,27(0,90)$ & $0,3629 * * * *$ \\
\hline Práticas de autocuidado & $2,40(1,48)$ & $2,19(1,40)$ & $0,5219 * *$ \\
\hline Seguimento após alta hospitalar & $1,95(1,62)$ & $2,72(1,73)$ & $0,0381 * *$ \\
\hline
\end{tabular}


de medicamentos, cuidados com a ferida cirúrgica, reconhecimento dos sinais e sintomas de rejeição e de efeitos colaterais dos imunossupressores, entre outros cuidados, os quais são informados pela equipe multidisciplinar durante a internação, na alta hospitalar e, reforçados nos retornos ambulatoriais. Essas alterações no padrão de vida, juntamente ao medo do insucesso do transplante, geram ansiedade e estresse, experimentados pelo receptor em seu ambiente familiar. O enfermeiro, em conjunto com as ações interdisciplinares, poderá desenvolver planos de ensino para auxiliar pacientes e familiares no enfrentamento dessas situações, contribuindo para reduzir o estresse, melhorando a autoestima, a adesão ao tratamento e o retorno progressivo às atividades cotidianas..$^{1-2,10}$

Os objetivos da educação, após o transplante, são fortalecer o processo E-A iniciado durante a espera pelo órgão e preparar o paciente e a família com conhecimento e habilidades para participarem ativamente na gestão dos cuidados de saúde em longo prazo. A equipe muldisciplinar deve estar preparada para esclarecer as dúvidas e preocupações, bem como adaptar as informações sobre os cuidados pós-transplante de acordo com o nível social, educacional e cultural dos pacientes. ${ }^{5}$ Os resultados da presente pesquisa reforçaram a relevância da equipe multidisciplinar como uma das principais fontes de informação sobre os cuidados domiciliares após o transplante.

Em relação às necessidades de informação, as complicações após o transplante foi a que obteve média maior $(5,35)$, seguida pelos medicamentos após a cirurgia $(5,29)$. Esses resultados são similares aos indicados em pesquisa realizada no mesmo centro transplantador, com candidatos ao transplante de fígado, na qual as principais necessidades de informação relatadas foram relacionadas às complicações e aos cuidados, após o transplante. ${ }^{4} \mathrm{Em}$ estudo desenvolvido na América do Norte, as questões relacionadas à qualidade de vida, ao uso de medicamentos e ao seguimento após o transplante, foram as lacunas de conhecimento identificadas entre os receptores de órgãos abdominais. ${ }^{8}$ Os dados mencionados apontam a preocupação dos pacientes, antes e após a intervenção cirúrgica, com o insucesso do transplante.

$\mathrm{Na}$ categoria manejo das potenciais complicações foram agrupadas seis necessidades de informação, com base na análise das médias de cada necessidade; pode-se inferir que os receptores indicaram que o aprendizado dos assuntos em questão é relevante na vida, após o transplante. Assim, reconhecer os sinais e sintomas, comparecer aos retornos ambulatoriais e realizar os exames regularmente podem prevenir
- aparecimento de infecções, rejeição, recidiva de tumores, recorrência da doença de base e efeitos secundários à imunossupressão.

Devido à doença que acarretou o transplante relacionarse ao estado nutricional e à necessidade de restrições alimentares, os resultados deste estudo indicaram interesse dos receptores sobre alimentação segura após o transplante, sendo a que recebeu o maior número de votos $(74)$ e a terceira maior média $(4,53)$. Observouse ainda que quando avaliado o IMC em relação ao tempo de transplante, houve diferença estatisticamente significante entre os grupos I e II (Tabela 3). Esses resultados indicaram média maior de IMC em pacientes com três anos ou mais de transplante, dado que corrobora a necessidade de atenção da equipe de saúde com o controle de peso, após o transplante de fígado.

Em longo prazo, um dos principais objetivos sociais do transplante de fígado é o retorno do indivíduo à população economicamente ativa e produtiva. Contudo, após o transplante, diferentes problemas emocionais afetam a pessoa, como a depressão, o medo da rejeição e a ansiedade, que reduzem com frequência a capacidade para o trabalho e o convívio social. ${ }^{710-11}$ Apesar de alguns pacientes considerarem o retorno às atividades laborais como indicativo de boa recuperação pós-transplante, algumas condições para o regresso ao trabalho ainda causam preocupação nos receptores, a saber: exigências físicas, risco de infecção graças à exposição a diferentes grupos de pessoas e à incapacidade de executar as atividades necessárias. Outra preocupação indicada pelos pacientes é a perda dos benefícios financeiros conquistados antes do transplante, no caso de insucesso no retorno ao trabalho..$^{12-13}$ No presente estudo, na categoria de questões sobre estilo de vida, foram agrupadas sete necessidades de informação, sendo que o retorno ao trabalho/estudo e as atividades sociais e de lazer, obtiveram médias mais baixas, indicando menor interesse dos receptores de transplante de fígado.

O transplante de fígado é um procedimento complexo, indicado para indivíduos com doença hepática grave, onde todas as alternativas terapêuticas já foram esgotadas. $\mathrm{Na}$ espera pelo transplante, os pacientes experimentam condições de estresse relacionadas ao estado físico e psicológico, agravadas pela doença e pela necessidade de aguardar um doador. Após o transplante, os receptores precisam aderir ao plano de tratamento complexo e contínuo que envolve a automedicação efetiva, dieta específica, acompanhamento ambulatorial frequente, mudanças no estilo de vida e autocuidado rigoroso, para que sejam atingidos bons resultados de 
sobrevida e qualidade de vida, sendo que o sucesso dos transplantes está diretamente relacionado à adesão ao tratamento pós-operatório proposto. ${ }^{14-15}$

A melhoria da qualidade de vida e a prevenção de complicações podem ser alcançadas quando o paciente conhece a proposta terapêutica e a importância do seguimento das informações, após o transplante. $O$ processo E-A é facilitado quando a equipe conhece o perfil social, econômico e cultural dos pacientes, ou seja, quais são as crenças, as dificuldades e as necessidades destes diante da condição de vida, cuidados e tratamento após a intervenção cirúrgica, em curto e longo prazo. ${ }^{11,16}$

Assim, o conhecimento das necessidades de informação consiste no primeiro passo para o desenvolvimento de um plano de ensino efetivo. Nesse contexto, o enfermeiro, enquanto membro da equipe multiprofissional especializada, tem papel importante no planejamento e na implementação de intervenções educativas capazes de estabelecer vínculos e aumentar a satisfação dos pacientes, proporcionando melhor adesão ao tratamento proposto.

Como limitação do estudo, ressalta-se o tamanho amostral e a generalização dos resultados, que deve ser cautelosa, uma vez que foram avaliados receptores de transplante de fígado de centro de referência do interior paulista.

\section{CONCLUSÃO}

Os resultados do estudo oferecem subsídios sobre o perfil sociodemográfico e clínico de receptores de transplante de fígado na realidade nacional, bem como sobre as necessidades de informação, que podem auxiliar o planejamento de intervenções educativas direcionadas para o processo de transplante de fígado, principalmente a adesão ao tratamento e a melhoria da qualidade de vida. Além disso, o conhecimento gerado pode auxiliar as equipes multiprofissionais especializadas em transplantes, em especial ao enfermeiro que tem papel de educador na saúde. Esse profissional pode desenvolver estratégias de E-A voltadas ao receptor de transplante de fígado, considerando as modificações no comportamento e estilo de vida destas pessoas. Devido à escassez de estudos nacionais sobre a temática investigada, os resultados também indicaram a necessidade de condução de novas pesquisas que poderão propor intervenções educativas que possam melhorar a qualidade da assistência, a adesão ao tratamento e a prevenção de complicações, após o transplante.

\section{ABSTRACT}

Purpose: Assessing the information needs to liver transplant recipients in outpatient hospital care. Method: Descriptive study conducted in a Brazilian transplant center. The sample consisted of 92 participants. Data collection was performed over a five-month period, with application of an adapted instrument. Results: The information needs that obtained the highest averages were: complications after transplantation (5.35), medications after surgery (5.29), and food intake after transplantation (4.53). The information needs that obtained lower averages were related to pregnancy and contraception (0.28), social and leisure activities (0.89), and return to work / study (0.97). Conclusion: The resulting knowledge on the information needs can help in planning educational interventions, which can improve the quality of care, treatment adherence and prevention of complications after transplantation.

Keywords: Organ Donors; Transplantation; Organ Donation; Students, Medical. 


\section{REFERÊNCIAS}

1. Dias FFM, Almeida SS, Almeida MD. Assessment and enrolment process for liver transplantation: nursing management through quality indicators. Einstein. 2018;16(1):eAO3975.

2. Oliveira RA, Turrini RN, Poveda Vde B. Adherence to immunosuppressive therapy following liver transplantation: an integrative review. Rev. Latino-Am. Enfermagem. 2016;24:e2778.

3. Sa AS, Ziviani LC, Castro ESO, Galvão CM, Mendes KD. Information needs of family caregivers regarding liver transplant candidates. Rev Gaúcha Enferm. 2016;37(1):e54650.

4. Mendes KD, Rossin FM, Ziviani Lda C, de Castro ESO, Galvão CM. Information needs of liver transplant candidates: the first step of the teaching-learning process. Rev Gaúcha Enferm. 2012;33(4):94-102.

5. Bernardina D, Phillips DK. Education for transplant patients and caregivers. In: Cupples S, Lerret S, McCalmont V, Ohler L, editors. Core Curriculum for Transplant Nurses. 2nd ed. Philadelphia: Wolters Kluwer; 2017. p. 50-85.

6. Bastable SB, Gramet P, Jacobs K, Sopczyk D. Health professional as educator: principles of peaching and learning. Sudbury, MA: Jones \& Bartlett Publishers; 2011. $628 \mathrm{p}$.

7. Oliveira PC, Mucci S, Silva ESV, Leite RF, Paglione HB, Erbs $\mathrm{JL}$, et al. Assessment of Factors Related to Adherence to Treatment in Liver Transplantation Candidates. Transplant Proc. 2016;48(7):2361-5.
8. Myers J, Pellino TA. Developing new ways to address learning needs of adult abdominal organ transplant recipients. Prog Transplant. 2009;19(2):160-6.

9. Serper M, Wolf MS. Is liver transplant education patientcentered? Liver Transplant. 2017;23(9):1233-4.

10. Teixeira HR, Marques DM, Lopes AR, Ziviani LC, Magro JT, Mente ED, et al. Anxiety and Stress Levels on Liver Transplantation Candidates. Transplant Proc. 2016;48(7):2333-7.

11. Burra P, Ferrarese A, Feltrin G. Quality of life and adherence in liver transplant recipients. Minerva Gastroenterol Dietol. 2018;64(2):180-6.

12. Mellinger JL, Volk ML. Transplantation for Alcoholrelated Liver Disease: Is It Fair? Alcohol Alcohol. 2018;53(2):173-7.

13. Kimbell B, Murray SA. What is the patient experience in advanced liver disease? A scoping review of the literature. BMJ Support Palliat Care. 2015;5(5):471-80.

14. Gillespie M, Rizzolo D. A systems-based approach to patient care after liver transplantation. JAAPA. 2018;31(1):14-9.

15. Grogan TA. Liver transplantation: issues and nursing care requirements. Crit Care Nurs Clin North Am. 2011;23(3):443-56.

16. Morales JM, Varo E, Lazaro P. Immunosuppressant treatment adherence, barriers to adherence and quality of life in renal and liver transplant recipients in Spain. Clin Transplant. 2012;26(2):369-76. 